\title{
Cognitive processess and cognitive reserve in multiple sclerosis
}

\author{
G. CHILLEMI ${ }^{*}$, C. SCALERA ${ }^{1 *}$, C. TERRANOVA ${ }^{1}$, A. CALAMUNERI ${ }^{1}$,

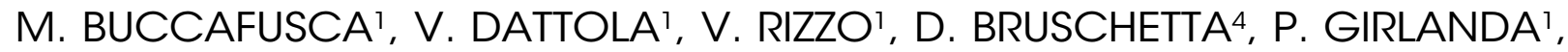 \\ A. QUARTARONE $1,2,3$ \\ ' Department of Neurosciences, University of Messina School of Medicine, Messina 98100, Italy; \\ ${ }^{2}$ Department of Physiology, Pharmacology \& Neuroscience, City University of New York Medical \\ School, New York, New York 10031; ${ }^{3}$ Istituto Di Ricovero e Cura a Carattere Scientifico (IRCCS), \\ Centro "Bonino Pulejo", Messina, Italy; ${ }^{4}$ Dipartimento di Scienze Biomediche e delle Immagini \\ Morfologiche e Funzionali \\ *The authors equally contributed to the present work.
}

\begin{abstract}
A B S T R A C T
Multiple Sclerosis (MS) is characterized by motor, cognitive, and neuropsychiatric symptoms, which can occur independently.

While MS is traditionally considered an inflammatory disease of the white matter, degeneration of gray matter is increasingly recognized as an important contributor to the progressive cognitive decline. A protective factor against the progression of cognitive dysfunction in MS could be the cognitive reserve, defined as resistance to brain dysfunction.

Aim of the present study is to evaluate the role of cognitive reserve for different aspects of cognitive dysfunction of patients with $M S$.

We found that patients with MS and lower cognitive reserve have poorer neuropsychological performance and slower information speed processing.

These findings support the notion that intellectual reserve may protect some aspects of cognitive function in patients with $M S$.
\end{abstract}

Key words

Multiple sclerosis $\bullet$ Cognitive Reserve $\bullet$ Verbal cognitive processes $\bullet$ Visual-space processes

\section{Introduction}

Multiple sclerosis (MS) is a progressive disease of the central nervous system characterized by the production of widespread lesions, or plaques, in the brain and spinal cord. Despite a growing number of publications related to cognition and MS, the precise nature of cognitive deficits, their frequency and their evolution throughout the disease process remains to be established. Cognitive impairment is a common clinical feature of MS, with a prevalence ranging from $43 \%$ to $70 \%$, occurring either at earlier and later stages of the disease (Pelosi et al., 1997; Piras et al., 2003).

MS may impact various aspects of cognitive functioning, including attention (Litvan et al., 1988), information processing efficiency (Diamond et al., 1997), executive functioning, processing speed, and long-term memory (Rao, 2004).

Processing speed, visual learning and memory seem to be the most commonly functions affected in MS. On the other hand, "simple" attention (e.g., repeating digits) and essential verbal skills (eg, word nam- 
ing and comprehension) are not usually affected. Although most studies indicate that general intelligence remains intact in patients with MS, other investigations have detected a slight but significant decrement (Amato et al., 2013). Overt dementia is rare in MS, while only single cognitive domains are known to be usually involved. (Benedict et al., 2010) The lack of correlation between the degree of brain pathology or brain damage and the cognitive symptoms strongly supports the idea that brain reserve is a powerful adaptive plastic mechanism compensating for brain damage (Stern, 2002). The reserve model theory postulates the presence of two different types of reserve: the first one called "brain reserve", which can be considered a passive process, and a second one defined as "cognitive reserve", which is the brain ability to cope with or compensate for pathology (Stern, 2002).

The reserve theory posits that both heritable/genetic (i.e. maximal lifetime brain growth, MLBG) (Scarmeas et al., 2003; MacLullich et al., 2002) and environmental factors (intellectual enrichment, Cognitive Reserve) contribute to protect against disease-related cognitive decline (Sumowski et al., 2010).

Many studies in the past linked MLBG to "brain reserve", hypothesizing that cognitive impairment/ dementia might emerge when total brain volume falls below a critical (albeit unspecified) threshold (Satz, 1993). Patients with larger MLBG might thus reach later critical volume threshold associated with cognitive impairment and dementia with respect to subjects with smaller MLBG (Feinstein et al., 2013). Consequently, persons with larger MLBG might better withstand neurologic disease burden (and associated brain volume loss/atrophy) without suffering from cognitive impairment/dementia (Sumowski et al., 2012).

Cognitive reserve (CR) postulates that a set of life experiences such as educational and occupational exposure and leisure activities are associated with reduced risk of developing dementia and with a slower rate of memory decline in normal aging, thus having an high impact in task performances involving cognitive processes. Studies on cognitive reserve in Alzheimer disease (AD) and MS have confirmed CR hypothesis, showing that intellectually enriching lifestyles may protect against cognitive decline (Sumowski et al., 2013). Aim of this study was to investigate which cognitive functions are preserved by $\mathrm{CR}$ in patients affected by MS.

\section{Methods}

Twenty-nine patients with definite MS without any exacerbation in the last 4 weeks were studied. Patients did not make use of corticosteroids at the time of the study, and have no history of serious psychiatric illness, substance abuse, learning disability, or other neurologic condition. Mean age was 43.7 years $(\mathrm{SD}=13.05)$, with 13.1 years of education $(\mathrm{SD}=2.5)$. Mean disease duration was 6.9 years $(\mathrm{SD}=1.7)$, with a mean Expanded Disability Status Scale score of 2.5 ( $\mathrm{SD}=0.8)$. MS phenotypes included relapsing-remitting and secondary progressive, patients had an average Mini Mental State Examination score of $28(\mathrm{SD}=3.5)$. For this study, we did not include a control group, but we rather wish to compare psychological outcomes of our MS group with standard scores available in related literature for each of psychological test administered to each patient. In table 1 we reported standard scores we used as benchmark, as well as average results from MS group.

\section{Standard protocol approvals and patients consent}

The present study was conducted in accordance with the ethical standards of the University of Messina, the local ethics committee, and the Declaration of Helsinki. Informed consent was obtained from all patients.

\section{Cognitive functioning}

Cognitive efficiency was measured with the Auditory Verbal Learning Test (RAVLT), the Rey-Osterrieth Complex Figure Test (ROCF), the Trail Making Test (TM), the Verbal Fluency Test (F) and the STROOP tests (STROOP).

RAVLT mainly evaluates short-term and long-term auditory-verbal memory functions. It consists of three test conditions: Immediate Recall (RAVLT - STM), Delayed Recall (RAVLT - LTM) and Recognition (RAVLT - R).

ROCF is a neuropsychological assessment, which evaluates short-term and long-term visual-spatial memory functions. It is characterized by three test conditions: Copy (ROCF- Copy), Immediate Recall (ROCF - STM) and Delayed Recall (ROCF - LTM). Trail Making Test is focused on visual attention and task switching. It consists of three conditions: Part A (TM A), Part B (TM B) and part BA (TM BA). 
Verbal fluency test is an indicator of executive functioning and linguistic skills that implies a retrieval of words in response to a stimulus that could be a letter (Phonemic Fluency, PF) or a category (Semantic Fluency, SF).

STROOP measures selective attention, cognitive flexibility and processing speed. It is divided into three parts: the Denomination (STROOP D), Reading (STROOP R) and Interference (STROOP I).

\section{Estimate of cognitive reserve: Cognitive Reserve Index questionnaire (CRla)}

CRIq is an instrument for comprehensive assessment and measurement of quantity of cognitive reserve accumulated by individuals throughout their lifespan.

\section{Statistical Analysis}

Non parametric Wilcoxon one-tail signed rank test was computed to test significant differences between psychological scores and benchmark values. Furthermore, Pearson's correlation coefficient $r$ was computed to evaluate linear correlations between CRI and other scores. For each analysis, null hypothesis was rejected when estimated p-value was lower than 0.05 .

\section{Results}

Detailed results for all tests are reported in Table I, together with normative benchmarks. Performances in ROCF as well as TM and SF were significantly lower in MS patients compared to normal benchmark values (ROCF copy: mean $=31.43, \mathrm{p}=0.000024$; ROCF LTM: mean=12.31, $\mathrm{p}=0.000090$; TM A: mean $=91.85, \mathrm{p}=0.000007$; TM B: mean $=159.59$, $\mathrm{p}=0.000003 ; \mathrm{SF}:$ mean $=51.68, \mathrm{p}=0.000005)$. RAVLT scores and PF one were not significantly different from standard values $(\mathrm{p}=0.55, \mathrm{p}=0.47$ and $\mathrm{p}=0.94$ respectively). Patients' accuracy scores to STROOP tests were excellent (STROOP D value: mean=99.25, SD=1.66; STROOP R: mean=100, $\mathrm{SD}=0.05$, STROOP I: mean=95.34, $\mathrm{SD}=1.27$ ); however they required higher amount of time to complete STROOP D and STROOP $R$ tasks if compared to standard timing scores (STROOP D: mean $=110.67 \mathrm{sec}, \mathrm{SD}=59.9 \mathrm{sec}, \mathrm{p}=0.000051$; STROOP R: mean=63.59 sec, $\mathrm{SD}=30.65 \mathrm{sec}$, $\mathrm{p}=0.002821$ ). There was no significant difference when comparing amount of time our patients needed to perform STROOP I test with respect to related timing reference (STROOP I: mean $=200.5 \mathrm{sec}$, $\mathrm{SD}=69.47 \mathrm{sec}, \mathrm{p}=0.062)$. CRIq score were not significantly different from standard one (mean $=93.68$, $\mathrm{SD}=13.37, \mathrm{p}=0.54$ ). When investigating correlations between Cognitive Reserve Index questionnaire (CRIq) and other tests we found a significant positive correlation between CRIq and RAVLT STM score ( $r=.444, p=0.018)$, as well as between CRIq and RAVLT LMT ( $\mathrm{r}=.401, \mathrm{p}=0.034)$; furthermore CRIq negatively correlated with STROOP R (Time) score $(\mathrm{r}=-.456, \mathrm{p}=0.017)$ and STROOP I (Time) $(\mathrm{r}=-$ $.566, \mathrm{p}=0.03)$. Related scatter plots are shown in Fig. 1. No significant correlations were found between age factor and CRIq as well as other mentioned scores.

\section{Discussion}

Previous studies demonstrated that deficits in working memory performances were more pronounced in individuals with MS as the disease progressed or in patients with greater overall cognitive impairment (Archibald and Fisk, 2000; DeLuca et al., 2004; Lengenfelder et al., 2003). Here we showed abnormalities of working memory even at early stage of MS; these abnormalities were not however coupled with a global cognitive decline. Indeed, RAVLT and PF tests did not reveal any verbal memory deficits nor phonetic fluency disorders, nor deficits in denomination and reading tasks. On the other hand the ROCF and SF scores showed that MS patients had visual-space memory deficits as well as semantic fluency and visual-space organization disorders. Baddeley proposed a working memory model that can be divided in central executive, phonological loop and visual-spatial sketchpad (Baddeley, 1992). The first one acts as a supervisor system and controls the flow of informations from and to the other two systems. The phonological loop is a specialized storage system for speech-based information; however, it does not have any capacity for controlling attention or decision-making. Visual-spatial sketchpad is responsible for holding visual and spatial informations for short periods of time; it can be used during thinking, remembering and processing tasks 

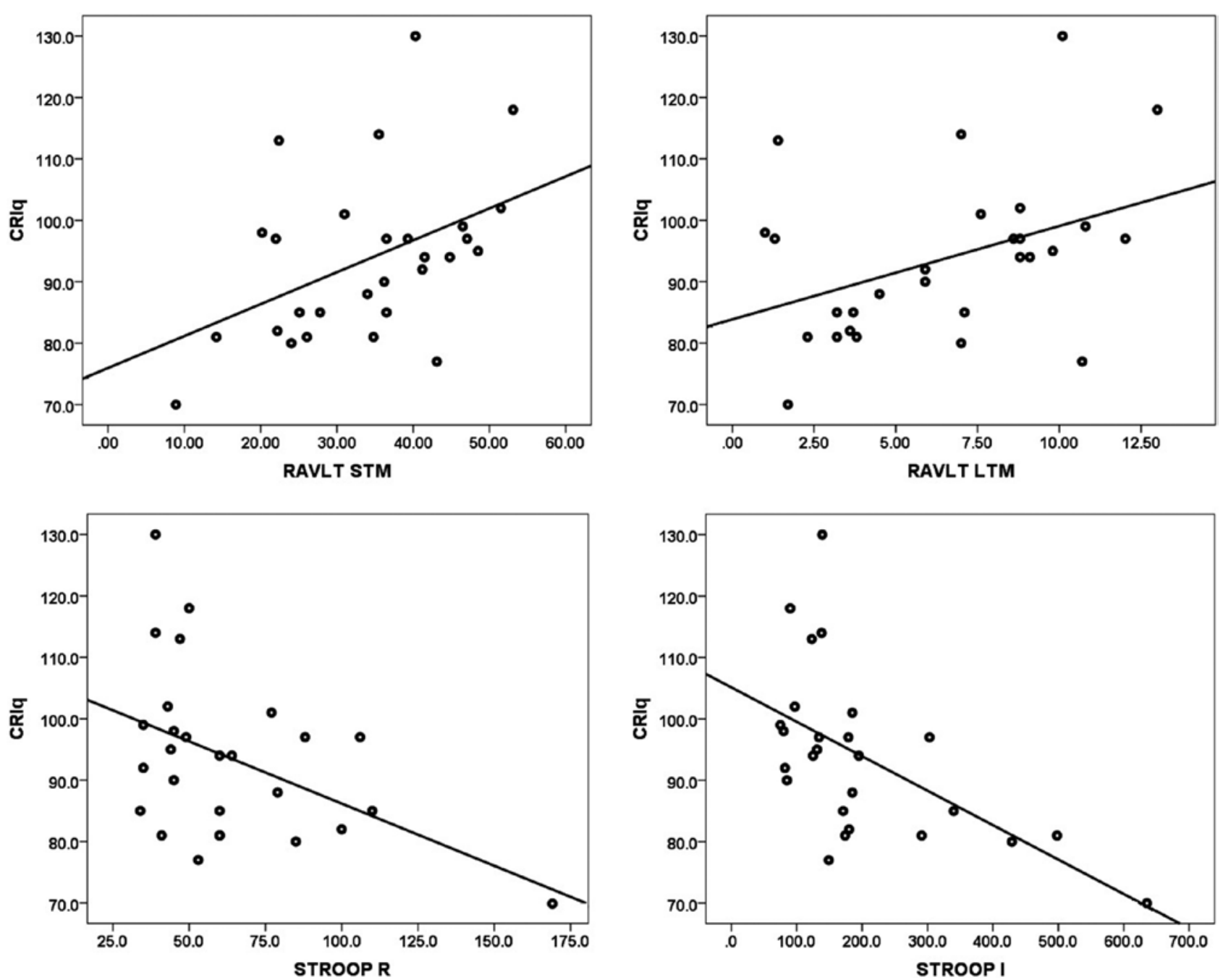

Fig. 1. - Significant correlations involving CRlq scores and other neuropsychological ones. Top-left panel reports scatter plot of RAVLT STM against CRIq. Top-right panel shows scatter plot of RAVLT LTM against CRlq. Bottom-left panel shows scatter plot of STROOP R (time) against CRlq. Bottom-right panel shows scatter plot of STROOP I (time) against CRla.

(Salway and Logie, 1995). ROCF tests are known to be related to visual-spatial domain, while RAVLT ones are linked to verbal domain. Since we found significant differences in ROCF but not in RAVLT in our MS group, in according with this model, we might hypothesize that visual-spatial domain is impaired in MS while this is not the case for the verbal one. Robinson and colleagues (2012) divided verbal fluency in "process selection" characterized by word retrieval from verbal memory and "process energization" which consists in production of new responses by visual-spatial cues. Selection is related to verbal cues, while energization is linked to visualspatial cues. In keeping with this distinction we could separate verbal component (PF) from visual- spatial one (SF), confirming again that impairment occur only at visual-spatial domain in MS.

Accuracy of STROOP tests was high in MS group even if achieved at the cost of longer reaction times in order to accomplish the tasks, as shown by increased reaction time in STROOP D and STROOP $\mathrm{R}$ tests. Albeit not significant, a similar trend was either found for STROOP I ( $\mathrm{p}=0.062)$. These results are in keeping with previous studies reporting that MS patients have deficits in processing speed when compared to healthy individuals (Demaree et al., 1999; Diamond et al., 1997).

Sumowski and colleagues demonstrated that earlylife intellectual enrichment protects MS patients from disease-related cognitive impairment (Sumowski et 
Table I. - Neuropsychological tests administered to the MS group. For each test, normative score as reported in literature is outlined, together with its standard deviation (if present). Average scores for our patients are shown, as well as standard deviations. Scores that were significantly different from normal ones are marked with an asterisk. Extended names of tests used are explained in details in the manuscript.

\begin{tabular}{|c|c|c|c|c|}
\hline \multirow{2}{*}{$\begin{array}{c}\text { TEST } \\
\text { RAVLT STM }\end{array}$} & \multicolumn{2}{|c|}{ Benchmark (SD) } & \multicolumn{2}{|c|}{ MS patients scores (SD) } \\
\hline & 34.29 & $(2.89)$ & 34.08 & $(11.42)$ \\
\hline RAVLT LTM & 6.47 & $(0.97)$ & 6.45 & $(3.53)$ \\
\hline ROCF copy* & 28 & $(0.25)$ & 31.43 & $(5.76)$ \\
\hline ROCF LTM* & 6.2 & $(2.75)$ & 12.31 & $(6.95)$ \\
\hline TM A* & 94 & NA & 91.85 & $(52.86)$ \\
\hline TM B* & 283 & NA & 159.59 & $(71.4)$ \\
\hline TM B-A & 187 & NA & 67.15 & $(31.93)$ \\
\hline $\mathrm{SF}^{*}$ & 35.5 & $(0.71)$ & 51.68 & $(14.81)$ \\
\hline PF & 25 & $(2.83)$ & 28.92 & $(11.66)$ \\
\hline STROOP D (seconds)* & 68.3 & NA & 110.67 & $(59.9)$ \\
\hline STROOP R (seconds)* & 45.6 & NA & 63.59 & $(30.65)$ \\
\hline STROOPI (seconds) & 147.4 & NA & 200.5 & $(69.47)$ \\
\hline CRI & 100 & NA & 93.68 & $(13.37)$ \\
\hline
\end{tabular}

al., 2009). Previous studies demonstrated that CR protects against the progression of cognitive dysfunction both in healthy individuals (Nucci et al., 2012), and in MS (Benedict et al., 2010).

The positive correlation between RAVLT and CRIq test might suggest that $\mathrm{CR}$ exert a protective role on verbal domain in MS patients. On the other hand the negative correlation between STROOP I and STROOP R (which are using visual cues) and CRIq may be explained considering $\mathrm{CR}$ is not directly involved in visual-spatial domain.

Furthermore, we did not find any significant correlation between CRIq scores and ROCFs copy test $(\mathrm{r}=.209, \mathrm{p}=0.286)$, Immediate Recall test $(\mathrm{r}=.375$, $\mathrm{p}=0.59)$, and Intermediate Recall test $(\mathrm{r}=.338, \mathrm{p}=0.84)$. Moreover, no significant correlations were found between CRIq scores and PF test $(\mathrm{r}=.132, \mathrm{p}=.502)$ and between CRIq and SF test $(r=.331, p=0.085)$. Altogether, these findings might indicate that visualspace functions are not influenced by CR.

In conclusion the significant correlation between $\mathrm{CR}$ and verbal domain process may suggest a positive association between cognitive reserve and verbal skills as proposed by the Baddeley's model of working memory. Since we detected no significant difference between standard scores and those of RAVLT tests, we hypothesized that CR have indeed a protective role on verbal component of working memory. On the other hand CR does not exert such protective role on the visual-spatial component of working memory.

The results of the present study may be relevant for conceiving future cognitive rehabilitation approaches aimed at improving the verbal component of working memory and the hence the quality life of MS patients.

\section{References}

Amato M.P., Langdon D., Montalban X., Benedict R.H., DeLuca J. Treatment of cognitive impairment in multiple sclerosis: position paper. $J$ Neurol., 260: 1452-68, 2013.

Archibald C.J. and Fisk J.D. Information processing efficiency in patients with multiple sclerosis. $J$. Clin. Exp. Neuropsychol., 22: 686-701, 2000.

Baddeley A. Working memory. Science, 255: 55659, 1992.

Benedict R.H., Morrow S.A., Weinstock Guttman B. Cognitive reserve moderates decline in information processing speed in multiple sclerosis patients. J. Int. Neuropsychol. Soc., 16: 829-835, 2010.

DeLuca J., Chelune G.J., Tulsky D.S., Lengenfelder J., Chiaravalloti N.D. Is speed of processing or working memory the primary information processing deficit in multiple sclerosis? J. Clin. Exp. Neuropsychol., 26: 550-62, 2004. 
Demaree H.A., DeLuca J., Gaudino E.A., Diamond B.J. Speed of information processing as a key defi cit in multiple sclerosis: Implications for rehabilitation. J. Neurol. Neurosurg. Psychiatry, 67: 661-663, 1999.

Diamond B.J., DeLuca J., Kim H., Kelley S.M. The question of disproportionate impairments in visual and auditory information in multiple sclerosis. $J$. Clin. Exp. Neuropsychol, 19: 34-42, 1997.

Feinstein A., Lapshin H., O'Connor P., Lanctôt K.L. Sub-threshold cognitive impairment in multiple sclerosis: The association with cognitive reserve. J. Neurol., 260: 2256-2261, 2013.

Lengenfelder J., Chiaravalloti N.D., Ricker J.H., DeLuca J. Deciphering components of impaired working memory in multiple sclerosis. Cogn Behav Neurol, 16: 28-39, 2003.

Litvan I., Grafman J., Vendrell P., Martinez J.M. Slowed information processing in multiple sclerosis. Arch. Neurol., 45: 281-85, 1988.

MacLullich A.M., Ferguson K.J., Deary I.J., Seckl J.R., Starr J.M., Wardlaw J.M. Intracranial capacity and brain volumes are associated with cognition in healthy elderly men. Neurology, 59: 16974, 2002.

Macniven J.A., Davis C., Ho M.Y., Bradshaw C.M., Szabadi E., Constantinescu C.S. STROOP performance in multiple sclerosis: information processing, selective attention, or executive functioning. $J$. Int. Neuropsychol. Soc., 14: 805-814, 2008.

Nucci M., Mapelli D., Mondini S. Cognitive Reserve Index questionnaire (CRIq): a new instrument for measuring cognitive reserve. Aging Clin. Exp. Res., 24: 218-226, 2012.

Pelosi L., Geesken J.M., Holly M., Hayward M., Blumhardt L.D. Working memory impairment in early multiple-sclerosis. Evidence from an eventrelated potential study of patients with clinically isolated myelopathy. Brain, 120: 2039-58, 1997.

Piras M.R., Magnano I., Canu E.D. Longitudinal study of cognitive dysfunction in multiple scle- rosis: neuropsychological, neuroradiological, and neurophysiological findings. J. Neurol. Neurosurg. Psychiatry, 74: 878-85, 2003.

Rao S.M. Cognitive function in patients with multiple sclerosis: impairment and treatment. Int. J. MS Care, 4: 9-22, 2004.

Robinson G., Shallice T., Marco Bozzali M., and Cipolotti L. The differing roles of the frontal cortex in fluency tests. Brain, 135: 2202-14, 2012

Salway A.F., Logie R.H. Visualspatial working memory, movement control and executive demands, $\mathrm{Br}$. J. Psychol., 86: 253-69, 1995.

Satz P. Brain reserve capacity on symptom onset after brain injury: A formulation and review of evidence for threshold theory. Neuropsychology, 7: 273-295,1993.

Scarmeas N., Zarahn E., Anderson K.E., Habeck C.G., Hilton J., Flynn J., Marder K.S., Bell K.L., Sackeim H.A., Van Heertum R.L., Moeller J.R., Stern Y. Association of life activities with cerebral blood flow in Alzheimer disease: implications for the cognitive reserve hypothesis. Arch. Neurol., 60: 359-365, 2003.

Stern Y. What is cognitive reserve? Theory and research application of the reserve concept. J. Int. Neuropsychol. Soc., 8: 448-460, 2002.

Sumowski J.F., Chiaravalloti N., DeLuca J. Cognitive reserve protects against cognitive dysfunction in multiple sclerosis. J. Clin. Exp. Neuropsychol., 31: 913-926, 2009.

Sumowski J.F., Wylie G.R., Chiaravalloti N. Intellectual enrichment lessens the effect of brain atrophy on learning and memory in multiple sclerosis. Neurology, 74: 1942-1945, 2010.

Sumowski J.F., Chiaravalloti N., Leavitt V.M. Cognitive reserve in secondary progressive multiple sclerosis. Mult. Scler., 18: 1454-1458, 2012.

Sumowski J.F., Rocca M.A., Leavitt V.M. Brain reserve and cognitive reserve in multiple sclerosis: What you've got and how you use it. Neurology, 80: 2186-2193, 2013. 